\title{
Hemodynamic features underlying pulmonary vein stump thrombus formation after left upper lobectomy: four-dimensional flow magnetic resonance imaging study
}

\author{
Tadashi Umehara ${ }^{1}$, Koji Takumi ${ }^{2}$, Kazuhiro Ueda ${ }^{1}$, Takuya Tokunaga ${ }^{1}$, Aya Harada-Takeda ${ }^{1}$, Masami Sato ${ }^{1}$ \\ ${ }^{1}$ Department of General Thoracic Surgery, Kagoshima University Graduate School of Medical and Dental Sciences, Kagoshima, Japan; ${ }^{2}$ Department \\ of Radiology, Kagoshima University Graduate School of Medical and Dental Sciences, Kagoshima, Japan
}

Contributions: (I) Conception and design: T Tokunaga, T Umehara, K Takumi, K Ueda, M Sato; (II) Administrative support: K Ueda; (III) Provision of study materials or patients: T Umehara; (IV) Collection and assembly of data: T Tokunaga, A Harada-Takeda, M Sato; (V) Data analysis and interpretation: T Tokunaga, T Umehara, K Takumi, K Ueda, M Sato; (VI) Manuscript writing: All authors; (VII) Final approval of manuscript: All authors.

Correspondence to: Kazuhiro Ueda, MD, PhD. Department of General Thoracic Surgery, Kagoshima University Graduate School of Medical and Dental Sciences, 8-35-1 Sakuragaoka, Kagoshima 890-8520, Japan. Email: k7433286@kadai.jp.

Background: We previously reported that to-and-fro blood movement near the pulmonary vein stump was particularly prominent after left upper lobectomy compared with other lobectomy, which may be the cause of the high susceptibility of pulmonary vein thrombus after left upper lobectomy. The aim of the present study was to compare the hemodynamics in patients who developed pulmonary vein thrombus after left upper lobectomy with those in patients who did not develop pulmonary vein thrombus using four-dimensional flow magnetic resonance imaging (4D MRI).

Methods: This was a retrospective evaluation of a prospectively collected clinical and radiological database of 37 patients who underwent 4D MRI 7 days after left upper lobectomy for lung cancer (n=37). We obtained two parameters by 4D MRI: the grade of to-and-fro blood movement and the flow energy loss around the pulmonary vein stump. The length of the pulmonary vein stump, a known risk factor for pulmonary vein thrombus, was also measured.

Results: According to a scatterplot of the grade of to-and-fro blood movement versus the flow energy loss, patients with pulmonary vein thrombus $(n=15)$ were concentrated in an area which appears to be a 'dangerous' hemodynamic condition. There were few patients without pulmonary vein thrombus in this 'dangerous' area, except for one who unfortunately developed delayed pulmonary vein thrombus and cerebral infarction. We proposed a formula using the 4D MRI-derived parameters based on a stepwise multiple regression analysis that was more closely associated with the development of pulmonary vein thrombus than the length of the pulmonary vein stump (area under the receiver operating characteristics curve: 0.918 vs. $0.705, \mathrm{P}=0.0500$ ).

Conclusions: We proposed the existence of a 'dangerous' hemodynamic condition responsible for pulmonary vein thrombus formation. 4D MRI before the development of pulmonary vein thrombus may help identify patients requiring preventive therapy against pulmonary vein thrombus and subsequent thromboembolic complications.

Keywords: Pulmonary vein stump thrombus; four-dimensional flow magnetic resonance imaging (4D MRI); left upper lobectomy (LUL); lung cancer

Submitted May 02, 2021. Accepted for publication Sep 13, 2021.

doi: 10.21037/qims-21-472

View this article at: https://dx.doi.org/10.21037/qims-21-472 


\section{Introduction}

Pulmonary vein stump thrombus after lung lobectomy is a critical adverse event that can cause vital organ embolism (1-5), although surgeons conventionally have paid little attention to this event. Ohtaka et al. noted the frequent occurrence of $\mathrm{PV}$ thrombus after left upper lobectomy (LUL) in 2012 (3). Thereafter, concordant results were reported by many Japanese investigators $(1,2,6-8)$. A longer pulmonary vein conduit remained after LUL than with other lobectomy procedures, which was considered to be a primary pathogenesis of PV thrombus after LUL $(1,3,4,6,7,8)$. Stapling of the PV with a stapler can also increase the risk of thrombus formation by generating blood turbulence around the PV stump (6). Unfortunately, PV thrombus after LUL still occurs in patients who undergo additional proximal ligation of the PV stump after LUL $(7,8)$, suggesting that a different pathogenesis is involved in the development of PV thrombus.

We previously evaluated regional hemodynamics by visualizing the blood streamlines in the left atrium from pulmonary veins as well as by the quantitative analysis of the blood flow around PV stump on four-dimensional flow magnetic resonance imaging (4D MRI) in patients who underwent lung lobectomy (9). We detected several characteristic blood flow patterns in the left atrium that were observed predominantly after LUL. In addition, according to the blood flow rate analysis, the to-and-fro blood movement was significantly more prominent around the PV stump after LUL than after other lobectomies. Although these results may suggest that extensive to-andfro blood movement, representing blood turbulence, around the PV stump is associated with thrombus formation after LUL, we were unable to identify the specific hemodynamic conditions likely to cause PV thrombus because we mainly analyzed patients without PV thrombus (38 of the 40 studied patients did not develop PV thrombus).

In the present study, we compared the hemodynamics in patients who developed PV thrombus after LUL to those in patients who did not. We also evaluated the blood flow energy loss, a representative hemodynamic parameter obtained from 4D MRI that is generally the result of flow separation, turbulence, surface friction, and flow attachment.

We present the following article in accordance with the STARD reporting checklist (available at https://dx.doi. org/10.21037/qims-21-472).

\section{Methods}

\section{Patients}

The present study included 37 patients who had undergone LUL for lung cancer with radical intent in our institution from November 2017 to August 2020. To exclude the effect of cardiac functional variability on the pulmonary venous flow, patients with the following conditions were excluded: ejection fraction $(\mathrm{EF})<50 \%$, grade $\geq$ III mitral valve regurgitation, significant asynergy in the ventricles, and atrial fibrillation. The study was approved by our institutional review board of No. 190286E, and informed consent was obtained from all of the patients. The study was conducted in accordance with the Declaration of Helsinki (as revised in 2013).

4D MRI was basically performed on postoperative day 7, along with the methodologies described below. The characteristics of the patients are shown in Table 1. Some patients had underlying diseases, such as hypertension and diabetes mellitus. However, no patients had preoperatively diagnosed blood coagulopathy or vascular endothelial dysfunction. Lobectomy was performed due to primary lung cancer $(\mathrm{n}=35)$ or metastatic lung tumor $(\mathrm{n}=2)$.

\section{Operation}

LUL was performed via 4 port sites $(n=25)$ or open thoracotomy $(n=12)$ under general anesthesia with endobronchial intubation. We used an endoscopic stapler (Ethicon, Cincinnati, OH, USA; or Covidien, Minneapolis, $\mathrm{MN}, \mathrm{USA}$ ) to divide the fused fissures as well as the pulmonary artery, PV, or bronchus. Proximal ligation of the left superior PV before stapling the left superior PV was performed in 25 patients. We basically performed upper mediastinal dissection for patients with primary lung cancer.

\section{MRI technique}

All magnetic resonance examinations were performed using 1.5- and 3-Tesla systems (Aera and Prisma, Siemens Healthcare, Erlangen, Germany) with a 30-channel body array coil. Prospective electrocardiography-gated 3D phasecontrast MRI with 3-directional velocity encoding was acquired using a coronal oblique volume covering the heart. The magnetic resonance scan parameters were as follows: flip angle, $8^{\circ}$; echo time (TE), $2.5-3.0 \mathrm{~ms}$; repetition time 
Table 1 Patient characters according to presence or absence of pulmonary vein thrombus

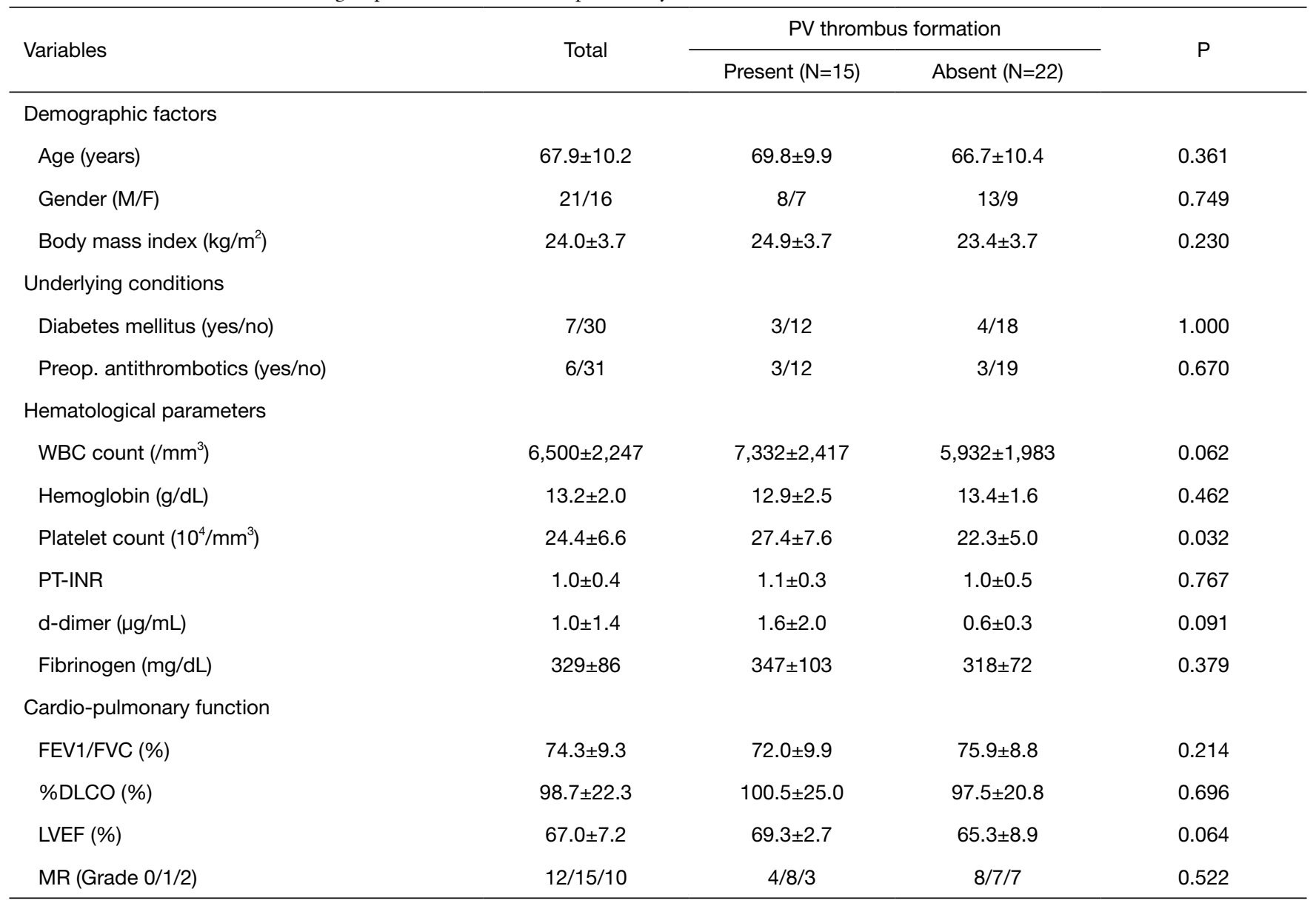

WBC, white blood cell; PT-INR, prothrombin time-international normalized ratio; FEV1/FVC, forced expiratory volume in 1 second to forced vital capacity ratio; DLCO, diffusing capacity of the lung for carbon monoxide; LVEF, left ventricular ejection fraction; MR, mitral valve regurgitation; PV, pulmonary vein.

(TR), $5.2 \mathrm{~ms}$; acceleration factor, 3; velocity encoding (VENC), $100 \mathrm{~cm} / \mathrm{s}$; field of view (FOV), $360 \mathrm{~mm} \times 360 \mathrm{~mm}$ spatial resolution, $2.0 \mathrm{~mm} \times 2.0 \mathrm{~mm} \times 2.5 \mathrm{~mm}$; phase, 10-15; and scan time, 11-20 s. To improve anatomical orientation, cine MR images in coronal views together with 4D flow data were acquired.

\section{Postprocessing}

For the calculation and visualization of the $3 \mathrm{D}$ velocity fields, 4D-flow and magnetic resonance angiographic data sets were analyzed using iTFlow (Cardio Flow Design Inc., Tokyo, Japan). The PV planes were defined orthogonal to the vessels at their junction with the left atrium (Figure 1). To visualize the atrial flow patterns, streamlines were emitted from each plane placed over the junction between
PV and left atrium (Figure 2, Video 1). We also evaluated the dynamic change in the flow rate at each PV plane in each patient by measuring the instantaneous flow rate at multiple time points during a single heartbeat (basically, 10 time points per patient) (Figure 2B,2C). The blood flow per single stroke in each PV was obtained by calculating the area under the flow rate-time curve (Figure 2B,2C). With regard to the left superior PV plane, according to our former study, the direction of the flow rate (antegrade $v s$. retrograde) changed dramatically, reflecting prominent toand-fro blood movement, as shown by a representative case in the current study (Figure 2A, Video 1). In order to grade the to-and-fro blood movement, we calculated the standard deviation (SD) of the instantaneous flow rate values in each patient (Figure 2B).

As the quantitative parameter to evaluate turbulent flow, 

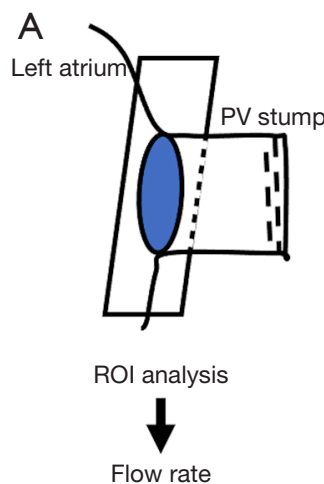

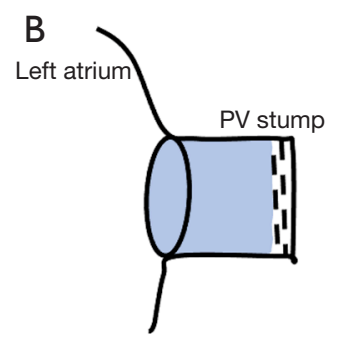

VOI analysis

Energy loss (EL)
Figure 1 Definition of the site of measurement in the blood flow rate and energy loss around the pulmonary vein stump. The region of interest (ROI) is placed at the junction between the pulmonary veins and left atrium to evaluate the blood flow rate (A). The volume of interest is placed at the residual left superior pulmonary venous lumen to evaluate energy loss (B). we calculated the energy loss (EL) defined by the following formula (10):

$$
E L=\int \sum_{i j} \frac{\mu}{2}\left(\frac{\partial u_{i}}{\partial x_{j}}+\frac{\partial u_{j}}{\partial x_{i}}\right)^{2} d v
$$

where $i$ and $j$ are coordinates in 2D, or 3D Euclidean space, $\mu$ is the blood viscosity, $x$ is the horizontal direction of the phase image, and $\mathrm{u}$ is the horizontal direction component of the blood velocity vector. This parameter indicates energy dissipation due to blood viscosity in turbulent flow. The kernel of the integral displays the distribution of the dissipated energy caused by the inefficient flow, which is integrated with the spatial increment dv. We measured the EL using the VOI placed in the PV stump after LUL (Figure 1B).

We also measured the length of the remaining venous conduit after lobectomy on oblique section images because the length of the remaining venous conduit is suspected to

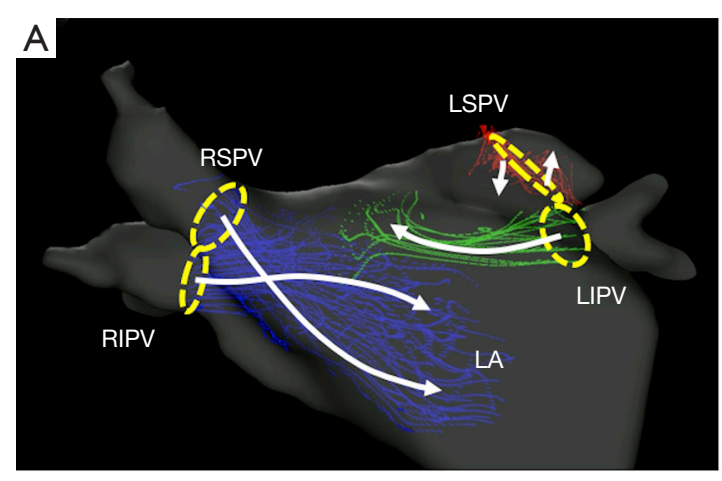

C

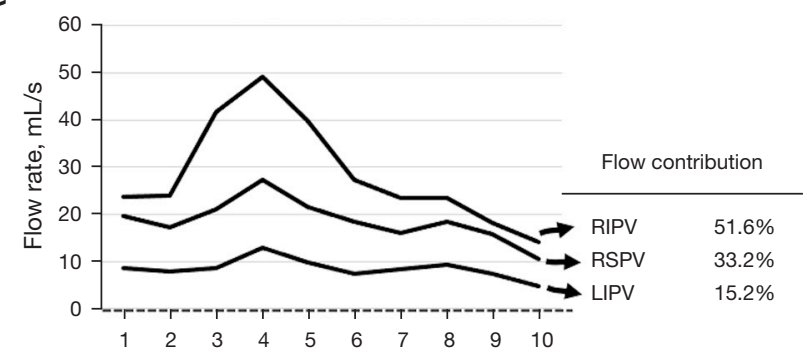

B

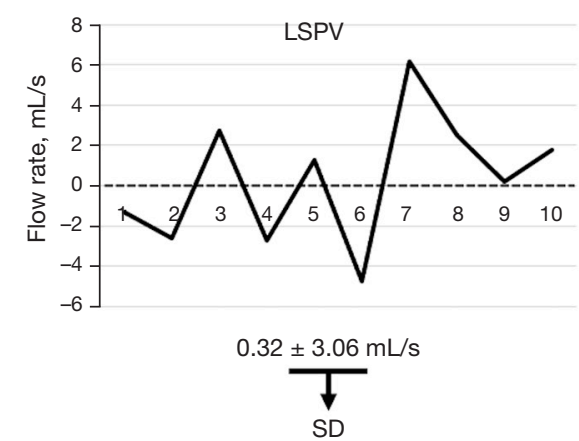

Figure 2 Four-dimensional flow magnetic resonance imaging findings in a 73-year-old man with pulmonary vein (PV) stump thrombus. The stream lines from the PV planes are shown in green, red, and blue (A). The instantaneous flow rates at 10 points during a single heartbeat in each PV plane are expressed by line graphs (B,C). The stream lines at the left superior PV junction (red lines) show to-and-fro blood movement (A), with findings compatible with those of the line graph showing the blood flow rate (B). The standard deviations (SDs) of the 10 instantaneous flow rate values at the left superior PV, being $3.2 \mathrm{~mL} / \mathrm{s}$, are used to grade to-and-fro movement. The flow contribution (\%) of each pulmonary vein can be calculated based on the line graphs (C). LSPV, left superior PV; LIPV, left inferior PV; RSPV, right superior PV; RIPV, right inferior PV. 


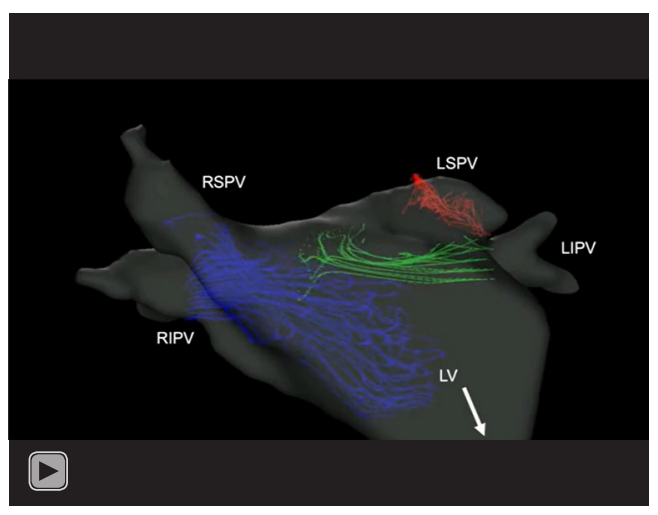

Video 1 A representative blood flow pattern in the left atrium on four-dimensional-flow magnetic resonance image in a patient who underwent left upper lobectomy. Blood flows from the right superior and inferior pulmonary vein (RSPV and RIPV) (blue lines) go smoothly to the left ventricle (LV). In contrast, blood flow from the left inferior pulmonary vein (RIPV) (green lines) goes cranially, forming an eddy stream in the atrium before going into the ventricle. Finally, to-and-fro static blood movement is found near the left superior pulmonary vein stump (red lines).

be associated with the risk of PV thrombus.

\section{The diagnosis of $P V$ thrombus}

PV stump thrombus were detected by the cine MRI images acquired together with $3 \mathrm{D}$ phase-contrast images. Cine MRI has been reported to be able to detect left atrial/left atrial appendage thrombus with high accuracy $(11,12)$. In addition, all PV thrombi were verified by enhanced computed tomography (CT) on the same day.

\section{Statistical analyses}

The values were expressed as the mean \pm standard deviation (SD). To compare patients with and without PV thrombus, an unpaired Student's $t$-test was used to test continuous variables of clinical and 4D flow parameters (SD and EL). Because PV thrombus predominantly develops under specific hemodynamic conditions with regard to SD and EL, we determined the median SD and median EL values in patients with $\mathrm{PV}$ thrombus. Based on the median values, we calculated the absolute difference between the SD or EL in each patient and the corresponding median values (ISD - median value I and IEL - median value I) to assess the risk of PV thrombus. Fisher's exact test was used to compare categorical variables. A stepwise multiple regression analysis was used to generate a regression equation for distinguishing patients with and without PV thrombus. The inputted outcome for the regression analysis was ' 1 ' for patients with PV thrombus and ' 0 ' for patients without PV thrombus. A receiver-operating characteristics (ROC) curve was constructed to determine the diagnostic performances of PV thrombus among various parameters by calculating the area under the ROC curve (AUC). P values of $<0.05$ were considered to indicate statistical significance. The statistical analyses were performed using the SPSS software program (SPSS statistics version 22; IBM, USA).

\section{Additional study}

To compare 4D MRI-derived parameters between LUL and other lobectomies, we evaluated an additional 27 patients who had undergone either right upper lobectomy $(n=11)$, right lower lobectomy $(n=8)$, or left lower lobectomy $(n=8)$ during the same study period. The technique used to evaluate the $4 \mathrm{D}$ flow parameters was the same as that used in the initial study. We compared the 4D parameters (SD and EL) between patients undergoing LUL and those undergoing other lobectomies using an unpaired Student's $t$-test.

\section{Results}

PV stump thrombus was identified in 15 (40.5\%) of the 37 patients. An antithrombotic drug was introduced in these 15 patients. Among them, one patient developed cerebral infarction on postoperative day 3 before identification of the PV thrombus. The thrombus eventually disappeared in all 15 patients after the introduction of antithrombotic drugs. Among the 22 patients without PV thrombus on postoperative day 7, 1 developed cerebral infarction and PV thrombus 5 months postoperatively.

Characteristic features according to the presence or absence of PV thrombus are shown in Table 1. The preoperative peripheral blood platelet count was significantly higher in patients who developed PV thrombus than in those who did not develop PV thrombus. The platelet count was above the upper normal limit $\left(>34.8 \times 10^{4} / \mathrm{mm}^{3}\right)$ in 4 patients, including 3 who developed PV thrombus and 1 who did not develop it $(\mathrm{P}=0.262)$. Likewise, the white blood cell count was above the upper normal limit $\left(>8,600 / \mathrm{mm}^{3}\right)$ in 3 patients, including 2 who developed PV thrombus and 1 who did not develop it $(\mathrm{P}=0.554)$. The $\mathrm{D}$-dimer 
Table 2 Surgical factors, oncological factors, and MRI-derived parameters according to the presence or absence of pulmonary vein thrombus

\begin{tabular}{|c|c|c|c|c|}
\hline Variables & Total & \multicolumn{2}{|c|}{ PV thrombus formation } & $\mathrm{P}$ \\
\hline \multicolumn{5}{|l|}{ Treatment factors } \\
\hline PV ligation (yes/no) & $25 / 12$ & $8 / 7$ & $17 / 5$ & 0.164 \\
\hline In-out balance, $\mathrm{mL} / \mathrm{m}^{2}$ & $811 \pm 446$ & $825 \pm 314$ & $802 \pm 524$ & 0.883 \\
\hline Histology type (ad/non-ad) & $28 / 9$ & $11 / 4$ & $17 / 5$ & 0.541 \\
\hline Solid tumor size (mm) & $18.1 \pm 11.0$ & $19.4 \pm 11.8$ & $17.2 \pm 10.7$ & 0.566 \\
\hline Nodal involvement (yes/no) & $7 / 30$ & $1 / 14$ & $6 / 16$ & 0.204 \\
\hline \multicolumn{5}{|l|}{ MRI-derived parameters } \\
\hline RSPV flow (\%) & $49.8 \pm 15.6$ & $50.0 \pm 12.9$ & $49.7 \pm 17.5$ & 0.952 \\
\hline LIPV flow (\%) & $18.3 \pm 9.3$ & $17.4 \pm 8.3$ & $18.9 \pm 10.1$ & 0.639 \\
\hline $\mathrm{SD}, \mathrm{mL} / \mathrm{s}$ & $3.27 \pm 1.63$ & $3.18 \pm 0.64$ & $3.32 \pm 2.06$ & 0.759 \\
\hline $\mathrm{EL}, 10^{-3} \mathrm{~mW}$ & $1.99 \pm 1.49$ & $1.23 \pm 0.61$ & $2.49 \pm 1.70$ & 0.004 \\
\hline$|\mathrm{SD}-3.290|, \mathrm{mL} / \mathrm{s}$ & $1.18 \pm 1.12$ & $0.52 \pm 0.37$ & $1.63 \pm 1.24$ & 0.001 \\
\hline$|\mathrm{EL}-1.147|, 10^{-3} \mathrm{~mW}$ & $1.06 \pm 1.33$ & $0.38 \pm 0.47$ & $1.53 \pm 1.53$ & 0.003 \\
\hline
\end{tabular}

PV, pulmonary vein; RIPV, right inferior PV; RSPV, right superior PV; LIPV, left inferior PV; MRI, magnetic resonance imaging; SD, standard deviation for blood flow rate; EL, energy loss.

level was above the upper normal limit $(>1.0 \mu \mathrm{g} / \mathrm{mL})$ in 6 patients, including 4 who developed PV thrombus and 2 who did not develop it $(\mathrm{P}=0.198)$.

Because postoperative dehydration can promote thrombus formation, we assessed intraoperative in-out fluid balance (Table 2) and found no significant difference in the intraoperative in-out fluid balance between patients with and without PV thrombus. With regard to the surgical procedure for $\mathrm{PV}$ dissection, $\mathrm{PV}$ thrombus developed in 8 of the $25(32 \%)$ patients who underwent PV dissection with additional proximal ligation and in 7 of the $12(58 \%)$ patients who did not undergo additional ligation $(\mathrm{P}=0.164)$ (Table 2). We did not observe significant differences in the oncological status (histological type, tumor size, and nodal involvement) between patients who did and did not develop PV thrombus (Table 2).

With regard to the MRI-based measurements, the length of remaining left superior PV conduit was significantly longer in patient with $\mathrm{PV}$ thrombus than in patients without (12.7 \pm 4.0 vs. 9.9 $93.3, \mathrm{P}=0.027$ ) (Table 2). According to the analysis of the blood flow at each PV junction (right superior PV, right inferior PV, and left inferior PV), the percentage of flow in each $\mathrm{PV}$ was not significantly different between patients with and without PV thrombus (Table 2). Figure $3 A$ shows a scatterplot of SD vs. EL. Although SD was not significantly different between the patients with and without PV thrombus (Table 2), the patients with PV thrombus were concentrated in an area (Figure $3 A$ ). The median value for SD and EL in patients with PV thrombus were 3.290 and 1.147 , respectively. Figure $3 B$ shows a scatterplot of ISD - 3.290| vs. IEL - 1.147।. Both ISD - 3.290। and IEL - 1.147। were significantly lower in patients with PV thrombus than in those without it (Table 2). According to a stepwise multiple regression analysis with covariates of the length of PV stump, ISD - 3.290।, and | EL - 1.147 I, ISD - 3.290 | and IEL - 1.147 I were selected as valid parameters to create a regression equation, as follows:

$$
\begin{aligned}
\text { Regression equation }= & 0.753-0.182 \times|\mathrm{SD}-3.290| \\
& -0.123 \times|\mathrm{EL}-1.147|
\end{aligned}
$$



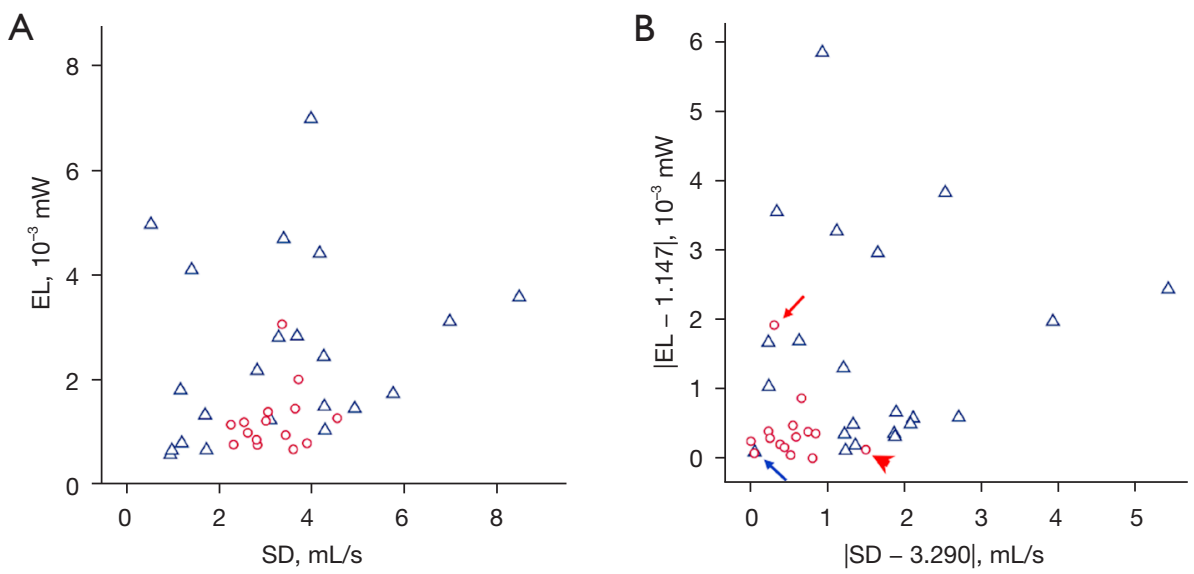

Figure 3 Exhibition of a relationship among the standard deviation of the flow rate at the left superior pulmonary venous junction (SD), energy loss (EL), and the presence or absence of pulmonary vein (PV) thrombus. Scatterplot of the SD vs. EL is shown in panel A. Data for patients with PV thrombus (red circle) were concentrated in a single area. The scatterplot is redrawn according to ISD - 3.290। $v s$. IEL 1.147 I (B) because the median values for SD and EL in patients with PV thrombus were 3.290 and 1.147, respectively. Although the data of patients with PV thrombus are concentrated near the origin of the coordinates, there were two outliers (red arrows) whose ISD - 3.290 I or IEL - 1.147 I exceeded the mean+2SD. One patient with a high ISD - 3.290 I (red arrow) had the highest preoperative platelet count $\left(43.2 \times 10^{4} / \mathrm{mm}^{3}\right)$ among all the patients, whereas another patient with a high IEL -1.147 I (red arrowhead) had the longest PV stump length $(19.2 \mathrm{~mm})$ among all of the patients. In contrast to the patients with PV thrombus, the data points of the patients without PV thrombus were located far from the origin of the coordinates (blue triangle), except for one patient (blue arrow) who patient developed delayed PV thrombus and cerebral infarction five months after the operation.

Table 3 Results of a receiver operating curve analysis for diagnosing pulmonary vein thrombus

\begin{tabular}{lcccc}
\hline Variables & Sensitivity, $\%$ & Specificity, $\%$ & Criterion & AUC \\
\hline Preop. PLT & 66.7 & 63.6 & $<23.4$ & 0.689 \\
PV stump length & 66.7 & 72.7 & $<11.0$ & 0.705 \\
$\mid$ EL $-1.147 \mid$ & 86.7 & 72.7 & $<0.472$ & 0.800 \\
$\mid$ SD $-3.290 \mid$ & 93.3 & 77.3 & $<0.842$ & 0.818 \\
Regression equation & 86.7 & 90.9 & $<0.227$ & 0.918 \\
\hline
\end{tabular}

Regression equation $=0.753-0.182 \times|S D-3.290|-0.123 \times|E L-1.147|$. AUC, area under the receiver operating curve; PV, pulmonary vein.

Table 3 shows the results of an ROC analysis for diagnosing PV thrombus. Although the AUC for PV stump length, IEL - 1.147।, and ISD - 3.290 I all exceeded 0.700, the AUC for the regression equation was the highest, besting the AUC for the PV stump length (AUC: $0.918 v s$. 0.705, $\mathrm{P}=0.0500$ ) (Figure 4).

Figure 5 shows the SD of the flow rate and the EL within the PV stump in each lobectomy group. The SD was significantly higher in patients undergoing LUL than in those undergoing other lobectomies. In contrast, the EL was not significantly different between patients undergoing LUL and those undergoing other lobectomies, except for patients undergoing left lower lobectomy.

\section{Discussion}

According to some literature, the overall incidence of cerebral infarction after lung cancer surgery is less than $1 \%$ $(13,14)$, which is comparable to that after general surgery $(15,16)$. However, when the analysis is restricted to patients undergoing LUL, the incidence of cerebral infarction markedly increases to $4.2 \%$ to $4.5 \%$, which is significantly higher than that observed after other types of lobectomy $(1,3)$. The increased incidence of cerebral infarction after LUL 


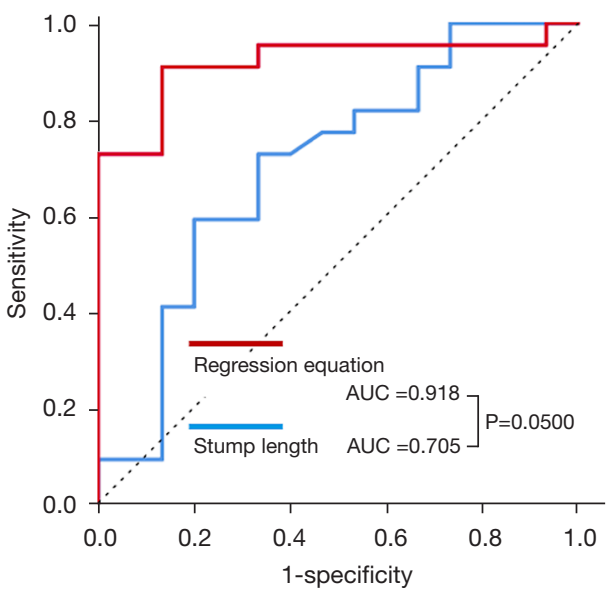

Figure 4 Receiver-operating characteristics (ROC) curves showing potential utility for diagnosing pulmonary vein (PV) thrombus. The area under the curve (AUC) for the regression equation, generated with the standard deviation (SD) and energy loss (EL), is higher than the AUC for the PV stump length (0.918 vs. 0.705, $\mathrm{P}=0.0500)$. Regression equation $=0.753-0.182 \times|\mathrm{SD}-3.290|-$ $0.123 \times|\mathrm{EL}-1.147|$.

was also verified by multicenter surveillance in Japan (17). These results may be due to the fact that PV thrombus develops almost exclusively after LUL (6).

According to a Japanese multicenter case-control study of 610 patients who developed cerebral infarction after lung cancer surgery, $31.7 \%, 42.8 \%$, and $47.8 \%$ of the patients developed cerebral infarction within 7,30, and 90 days after surgery, respectively (17). As expected, cerebral infarction developed predominantly after LUL at any time point (17). Therefore, we believe that patients who underwent LUL represents hemodynamic condition that likely contributes to development of PV thrombus at both early- and latepostoperative period, which was supported by the previous report on patients who developed PV thrombus late after surgery (more than 90 days later) $(6,18-20)$. In the present study, we hypothesized that the median SD and EL values in patients with $\mathrm{PV}$ thrombus reflected the ideal hemodynamic condition for PV thrombus formation. As expected, patients with PV thrombus were concentrated near the median SD and the median EL in the scatterplot. Using the SD, $\mathrm{EL}$, and their median values, we proposed a formula based on the results of a stepwise multiple regression analysis that proved more useful than the PV stump length for distinguishing patients with PV thrombus from those without it. We expect that early postoperative 4D MRI
A

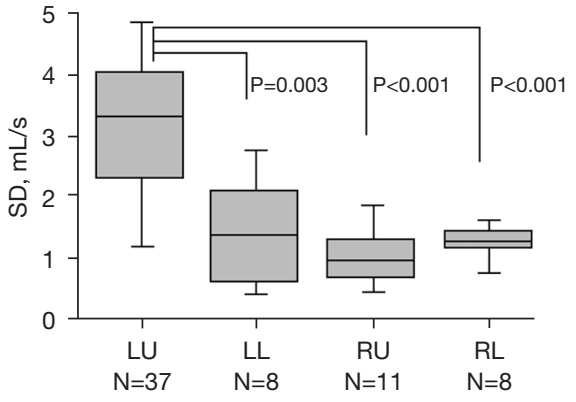

B

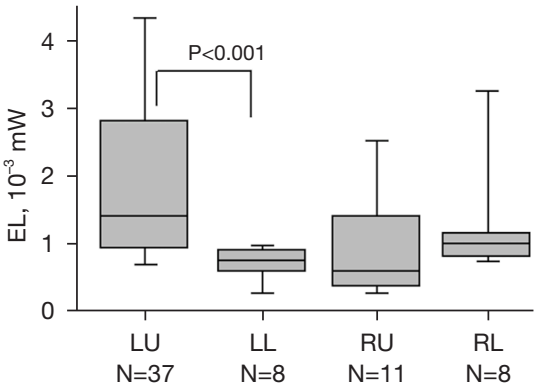

Figure 5 The standard deviation (SD) of the flow rate and the energy loss (EL) within the PV stump in each lobectomy group based on our database of patients who underwent four-dimensional flow magnetic resonance imaging on postoperatively day 7 . The SD is significantly higher in patients undergoing left upper lobectomy than in those undergoing other lobectomies (A). In contrast, the energy loss (EL) is not significantly different between patients undergoing left upper lobectomy and those undergoing other lobectomies, except for patients undergoing left lower lobectomy (B). LU, left upper; LL, left lower; RU, right upper; $\mathrm{RL}$, right lower.

may be useful for identifying patients requiring preventive therapy against PV thrombus after LUL. However, further hemodynamic study in late-postoperative period should be performed to verify our results.

We previously showed that the SD of the flow rate, representing the grade of to-and-fro blood movement, was significantly correlated with the length of the PV stump (9). We also showed that both the SD and PV stump length were significantly greater in patients who underwent LUL than in those who underwent other lobectomies, which seemed to contribute to the high incidence of PV thrombus after LUL. Therefore, we suspected that additional proximal ligation of the $\mathrm{PV}$ stump contributes to the reduction in the length of the PV stump, thereby reducing the $\mathrm{SD}$ value and consequently the incidence of $\mathrm{PV}$ 
thrombus after LUL. Unfortunately, in the current study, additional proximal ligation was not significantly associated with a reduction in the rate of $\mathrm{PV}$ thrombus formation. We found that although a longer PV stump and higher SD of the flow rate were predominantly observed after LUL, a higher SD was not associated with an increased risk of PV thrombus formation after LUL.

According to the quantitative analysis of the blood flow at each PV junction, the flow contribution was highest at the right superior $\mathrm{PV}$, followed by the right inferior $\mathrm{PV}$ and left inferior PV (Table 2). However, these disproportionate blood flow patterns were observed in both patients with and without PV thrombus formation. We believe that the hemodynamics within the left superior PV stump may be highly dependent on the location and geometry of each PV and the left atrium, rather than on the flow contribution of each PV. Therefore, the significance of the geometry of the PVs and left atrium after LUL for generating a 'dangerous' hemodynamic condition at the PV stump should be clarified by computational fluid dynamics technology. Of note, previous in vitro experimental studies have already demonstrated the agreement of MRI-derived velocity data with computational fluid dynamic models (20).

The visual observations of the streamlines at the junction between the left superior PV and the left atrium revealed the flow pattern to be very complex, consisting of multidirectional paths (see the videoclip) that may not be adequately graded by the SD of the flow rate alone. Therefore, we evaluated the EL in the current study. The EL of the flow is caused by the loss of power, such as when passing through complicated units $(21,22)$. The EL is generally induced by flow separation, turbulence, surface friction, and flow attachment. The energy lost in this manner may be transformed into other types of energy, such as heat and energy that wears away the vascular inner surfaces. Of note, the clinical usefulness of EL has been validated for estimating the outcomes of cardiovascular surgery $(23,24)$.

Based on Virchow's triad, blood flow stasis is an important contributing factor to venous thrombus formation (25), as is also the case with left atrial thrombus (26-29). However, blood flow stasis (low SD and low EL) was more predominantly observed after surgeries other than LUL (as shown in Figure 5) which did not cause PV thrombus. Since PV thrombus developed exclusively after LUL, some elevation of SD and EL may be needed to develop PV thrombus. In contrast, PV thrombus did not develop in patients with highest SD or highest EL, probably because highest SD or highest EL means active blood movement within the PV stump cavity, resulting in wash out of the stump cavity. Therefore, we hypothesized that some specific hemodynamic condition, regarding SD and $\mathrm{EL}$, is associated with the development of PV thrombus: we provisionally defined the optimal hemodynamic condition based on the median values for SD and EL in patients who developed PV thrombus.

The scatterplot of ISD - 3.290| vs. IEL - 1.147 | contributed considerably to distinguishing patients with PV thrombus from those without it, as the patients with PV thrombus were concentrated near the origin of coordinate. However, there were two outliers whose ISD - 3.290I or IEL -1.147 I exceeded the mean+2SD (as suggested by the red arrows in Figure 3B). One patient with a high ISD - 3.290। (red arrow) had the highest preoperative platelet count $\left(43.2 \times 10^{4} / \mathrm{mm}^{3}\right)$ among all the patients, whereas another patient with a high IEL - 1.147| (red arrowhead) had the longest PV stump length $(19.2 \mathrm{~mm})$ among all of the patients. Although the preoperative platelet count and PV stump length were not selected as valuable parameters for predicting $\mathrm{PV}$ thrombus based on multiple regression and ROC analyses, respectively, some patients with suprathreshold values for the platelet count and PV stump length may need to be excluded from the hemodynamicsbased risk assessment of $\mathrm{PV}$ thrombus.

In contrast to the patients with $\mathrm{PV}$ thrombus, the data points of the patients without PV thrombus were located far from the origin of the coordinates (Figure 3B), except for one patient (blue arrow) who developed delayed PV thrombus and cerebral infarction five months after the operation. These results suggest the usefulness of our strategy for assessing the risk of PV thrombus and identifying patients who need preventive therapy against PV thrombus. A large-scale study will further clarify this issue.

There are possible limitations in this study. First, we aimed to clarify the underlying hemodynamic features in PV thrombus formation. However, because we performed 4D MRI once postoperatively (on postoperative day 7), we could overlook patients who subsequently developed PV thrombus. Next, we proposed 'dangerous' hemodynamic condition based on flow velocity data in patients who have $\mathrm{PV}$ thrombus. Thus, concern remains if the presence of PV thrombus prevented to estimate real 'dangerous' hemodynamic condition. However, during the ROI placement for measurement of blood flow velocity at the PV junction, the ROI was not adjacent to the PV thrombus: the proportion of thrombus volume to PV stump volume 
was $19.2 \% \pm 10.9 \%(3.6-41.1 \%)$. In addition, the thrombus was not included within the VOI for determining the EL. Nonetheless, the influence of the presence or absence of PV thrombus within the PV stump on the blood flow velocity values at $\mathrm{PV}$ junction should be evaluated by computational fluid dynamics technology. Secondary, most of the papers on postoperative PV thrombus were reported from Japan (1-4,6-8). Thus, the incidence of this complication in different countries and different races remains unknown. Accumulation of the data from patients who underwent postoperative enhanced CT may be helpful to clarify this issue. In addition, multicenter case-control study of patients who developed cerebral infarction after lung lobectomy may be useful to clarify the risk of cerebral infarction after left upper lobectomy. Finally, according to previous reports, PV thrombus can also develop after left upper division segmentectomy (30,31), suggesting that left upper division segmentectomy may have increased risk of $\mathrm{PV}$ thrombus. Unfortunately, it remains unknown if patients who underwent left upper division segmentectomy show 'dangerous' hemodynamic condition on 4D MRI or not remains unclear because we have only a limited sample size.

\section{Conclusions}

We proposed the existence of a 'dangerous' hemodynamic condition responsible for PV thrombus formation after LUL using 4D MRI. 4D MRI before the development of PV thrombus may help identify patients requiring preventive therapy against PV thrombus after LUL. However, presently, postoperative screening of $\mathrm{PV}$ thrombus by enhanced CT is not mandatory because the role of preventive therapy in patients with $\mathrm{PV}$ thrombus on reducing the incidence of cerebral infarction will be clarified after large-scale randomized study.

\section{Acknowledgments}

Funding: This work was supported by grant-in-aid for scientific research JSPS KAKENHI (JP19K18221).

\section{Footnote}

Reporting Checklist: The authors have completed the STARD reporting checklist. Available at https://dx.doi. org/10.21037/qims-21-472

Conflicts of Interest: All authors have completed the ICMJE uniform disclosure form (available at https://dx.doi. org/10.21037/qims-21-472). TU reports that this study is supported by grant-in-aid for scientific research JSPS KAKENHI (JP19K18221) which is paid to his institution. The other authors have no conflicts of interest to declare.

Ethical Statement: The authors are accountable for all aspects of the work in ensuring that questions related to the accuracy or integrity of any part of the work are appropriately investigated and resolved. The study was conducted in accordance with the Declaration of Helsinki (as revised in 2013). The study was approved by our institutional review board (approval No. 190286E), and informed consent was obtained from all of the patients.

Open Access Statement: This is an Open Access article distributed in accordance with the Creative Commons Attribution-NonCommercial-NoDerivs 4.0 International License (CC BY-NC-ND 4.0), which permits the noncommercial replication and distribution of the article with the strict proviso that no changes or edits are made and the original work is properly cited (including links to both the formal publication through the relevant DOI and the license). See: https://creativecommons.org/licenses/by-nc-nd/4.0/.

\section{References}

1. Yamamoto T, Suzuki H, Nagato K, Nakajima T, Iwata T, Yoshida S, Yoshino I. Is left upper lobectomy for lung cancer a risk factor for cerebral infarction? Surg Today 2016;46:780-4.

2. Hattori A, Takamochi K, Kitamura Y, Matsunaga T, Suzuki K, Oh S, Suzuki K. Risk factor analysis of cerebral infarction and clinicopathological characteristics of left upper pulmonary vein stump thrombus after lobectomy. Gen Thorac Cardiovasc Surg 2019;67:247-53.

3. Ohtaka K, Hida Y, Kaga K, Iimura Y, Shiina N, Muto J, Hirano S. Pulmonary vein thrombosis after video-assisted thoracoscopic left upper lobectomy. J Thorac Cardiovasc Surg 2012;143:e3-5.

4. Nagaoka E, Yano M, Sugano T, Miyamoto T. Thrombus in the left superior pulmonary vein after left upper pulmonary lobectomy. J Thorac Cardiovasc Surg 2008;135:709-10.

5. Gual-Capllonch F, Teis A, Palomeras E. Pulmonary vein spontaneous echocontrast and stroke after pulmonary lobectomy. J Clin Ultrasound 2013;41:321-2.

6. Ohtaka K, Hida Y, Kaga K, Kato T, Muto J, Nakada- 
Kubota R, Sasaki T, Matsui Y. Thrombosis in the pulmonary vein stump after left upper lobectomy as a possible cause of cerebral infarction. Ann Thorac Surg 2013;95:1924-8

7. Nakano T, Kaneda H, Kawaura T, Kitawaki T, Murakawa T. Ligating the pulmonary vein at the pericardial reflection is useful for preventing thrombus formation in the pulmonary vein stump after left upper lobectomy. Gen Thorac Cardiovasc Surg 2019;67:450-6.

8. Miyoshi R, Nishikawa S, Tamari S, Noguchi M, Hijiya K, Chihara K. Pulmonary vein thrombosis after lobectomy with vein stump closure by ligation. Asian Cardiovasc Thorac Ann 2018;26:546-51.

9. Umehara T, Takumi K, Ueda K, Tokunaga T, HaradaTakeda A, Suzuki S, Sato M. Four-dimensional flow magnetic resonance imaging study to explain high prevalence of pulmonary vein stump thrombus after left upper lobectomy. J Thorac Dis 2020;12:5542-51.

10. Nakaji K, Itatani K, Tamaki N, Morichi H, Nakanishi N, Takigami M, Yamagishi M, Yaku H, Yamada K. Assessment of biventricular hemodynamics and energy dynamics using lumen-tracking 4D MRI without contrast medium. J Cardiol 2021;78:79-87.

11. Chen J, Zhang H, Zhu D, Wang Y, Byanju S, Liao M. Cardiac MRI for detecting left atrial/left atrial appendage thrombus in patients with atrial fibrillation : Meta-analysis and systematic review. Herz 2019;44:390-7.

12. Barkhausen J, Hunold P, Eggebrecht H, Schüler WO, Sabin GV, Erbel R, Debatin JF. Detection and characterization of intracardiac thrombi on MR imaging. AJR Am J Roentgenol 2002;179:1539-44.

13. Matsumoto K, Sato S, Okumura M, Niwa H, Hida Y, Kaga K, Date H, Nakajima J, Usuda J, Suzuki M, Souma T, Tsuchida M, Miyata Y, Nagayasu T. Frequency of cerebral infarction after pulmonary resection: a multicenter, retrospective study in Japan. Surg Today 2018;48:571-2.

14. Nojiri T, Inoue M, Takeuchi $Y$, Maeda $H$, Shintani Y, Sawabata N, Hamasaki T, Okumura M. Impact of cardiopulmonary complications of lung cancer surgery on long-term outcomes. Surg Today 2015;45:740-5.

15. Limburg M, Wijdicks EF, Li H. Ischemic stroke after surgical procedures: clinical features, neuroimaging, and risk factors. Neurology 1998;50:895-901.

16. Kam PC, Calcroft RM. Peri-operative stroke in general surgical patients. Anaesthesia 1997;52:879-83.

17. Matsumoto K, Sato S, Okumura M, Niwa H, Hida Y, Kaga K, Date H, Nakajima J, Usuda J, Suzuki M, Souma T, Tsuchida M, Miyata Y, Takeshi N; Committee for Patient
Safety, Quality Management of Japanese Association for Chest Surgery. Left upper lobectomy is a risk factor for cerebral infarction after pulmonary resection: a multicentre, retrospective, case-control study in Japan. Surg Today 2020;50:1383-92.

18. Usui G, Matsumoto J, Hashimoto H, Katano T, Kusakabe M, Horiuchi H, Okubo S. Thrombus Reformation in the Pulmonary Vein Stump Confirmed 16 Months After Cerebral Embolism on the Day After Left Upper Lobectomy for Lung Cancer. J Stroke Cerebrovasc Dis 2018;27:e225-7.

19. Yoon HJ, Kim KH, Jeong MH, Cho JG, Park JC. Very late unusual thrombosis of the remnant pulmonary vasculatures after lung resection complicated by embolic events. J Cardiothorac Surg 2019;14:196.

20. Venkatachari AK, Halliburton SS, Setser RM, White $\mathrm{RD}$, Chatzimavroudis GP. Noninvasive quantification of fluid mechanical energy losses in the total cavopulmonary connection with magnetic resonance phase velocity mapping. Magn Reson Imaging 2007;25:101-9.

21. Qian Y, Takao H, Umezu M, Murayama Y. Risk analysis of unruptured aneurysms using computational fluid dynamics technology: preliminary results. AJNR Am J Neuroradiol 2011;32:1948-55.

22. Barker AJ, van Ooij P, Bandi K, Garcia J, Albaghdadi M, McCarthy P, Bonow RO, Carr J, Collins J, Malaisrie SC, Markl M. Viscous energy loss in the presence of abnormal aortic flow. Magn Reson Med 2014;72:620-8.

23. Tsukui H, Shinke M, Park YK, Yamazaki K. Longer coronary anastomosis provides lower energy loss in coronary artery bypass grafting. Heart Vessels 2017;32:83-9.

24. Kamphuis VP, Elbaz MSM, van den Boogaard PJ, Kroft LJM, van der Geest RJ, de Roos A, Helbing WA, Blom NA, Westenberg JJM, Roest AAW. Disproportionate intraventricular viscous energy loss in Fontan patients: analysis by 4D MRI. Eur Heart J Cardiovasc Imaging 2019;20:323-33.

25. Brotman DJ, Deitcher SR, Lip GY, Matzdorff AC. Virchow's triad revisited. South Med J 2004;97:213-4.

26. Garcia J, Sheitt H, Bristow MS, Lydell C, Howarth AG, Heydari B, Prato FS, Drangova M, Thornhill RE, Nery P, Wilton SB, Skanes A, White JA. Left atrial vortex size and velocity distributions by 4D MRI in patients with paroxysmal atrial fibrillation: Associations with age and CHA2 DS2 -VASc risk score. J Magn Reson Imaging 2020;51:871-84.

27. Lee DC, Markl M, Ng J, Carr M, Benefield B, Carr JC, 
Goldberger JJ. Three-dimensional left atrial blood flow characteristics in patients with atrial fibrillation assessed by 4D flow CMR. Eur Heart J Cardiovasc Imaging 2016;17:1259-68.

28. Markl M, Lee DC, Ng J, Carr M, Carr J, Goldberger JJ. Left Atrial 4-Dimensional Flow Magnetic Resonance Imaging: Stasis and Velocity Mapping in Patients With Atrial Fibrillation. Invest Radiol 2016;51:147-54.

29. Markl M, Lee DC, Furiasse N, Carr M, Foucar C, Ng J, Carr J, Goldberger JJ. Left Atrial and Left Atrial

Cite this article as: Umehara T, Takumi K, Ueda K, Tokunaga T, Harada-Takeda A, Sato M. Hemodynamic features underlying pulmonary vein stump thrombus formation after left upper lobectomy: four-dimensional flow magnetic resonance imaging study. Quant Imaging Med Surg 2022;12(2):992-1003. doi: 10.21037/qims-21-472
Appendage 4D Blood Flow Dynamics in Atrial Fibrillation. Circ Cardiovasc Imaging 2016;9:e004984.

30. Asai K, Mochizuki T, Iizuka S, Momiki S, Suzuki K. Pulmonary vein stump thrombus: an early complication following upper division segmentectomy of the left lung. Gen Thorac Cardiovasc Surg 2014;62:244-7.

31. Kao CN, Liu YW. Acute cerebral infarction caused by atrial thrombus originating from left upper pulmonary vein stump after left upper lobe trisegmentectomy. Gen Thorac Cardiovasc Surg 2020;68:206-7. 